\title{
ESTUDO VOLTAMÉTRICO E ESPECTROFOTOMÉTRICO DO COMPLEXO CU(II)-GLIFOSATO
}

\author{
CLÁUDIA F. B. COUTINHO*
}

LUIZ H. MAZO**

O objetivo deste trabalho foi estudar o comportamento voltamétrico e espectrofotométrico do complexo $\mathrm{Cu}(\mathrm{II})$-glifosato em solução. Esse complexo apresentou duas bandas de absorção na região ultravioleta-visível (200 a 1000 nm): uma em 231 nm (transferência de carga) e outra em 731 nm (transição d-d). No estudo voltamétrico, utilizando a voltametria de onda quadrada (SWV), observou-se que a presença de glifosato deslocou o potencial de pico do $\mathrm{Cu}(\mathrm{II})$ de $+0,034 \mathrm{~V}$ para valor de potencial mais negativo $(-0,200 \mathrm{~V})$. A partir desses valores de potencial pode-se estimar a constante de estabilidade condicional do complexo igual a $\log K=7,9$. Os resultados obtidos neste artigo mostraram que 0 glifosato pode ser detectado na forma de complexo metálico, sem derivatização. O uso de metodologias de análise direta é muito interessante, devido a economia de reagentes e de tempo no preparo das amostras. A habilidade do glifosato em complexar grande variedade de metais pode ser utilizada com propósitos analíticos, como o desenvolvimento de metodologias de análise (voltamétrica e espectrofotométrica) desse herbicida em amostras de solo e água.

PALAVRAS-CHAVE: CU(II); GLIFOSATO; VOLTAMETRIA; ESPECTROFOTOMETRIA.

* Doutoranda em Química Analítica, Instituto de Química de São Carlos (IQSC), São Carlos, SP (e-mail: claudiabreda@iqsc.usp.br).

** Pesquisador, IQSC, São Carlos, SP (e-mail: Ihmazo@iqsc.usp.br). 


\section{INTRODUÇÃO}

O glifosato [N-(fosfonometil)glicina] é um herbicida sistêmico, pós-emergente e não-seletivo, largamente utilizado na agricultura (COUTINHO et al., 2005). O glifosato pertence ao grupo químico dos aminoácidos fosfonados e tal como seu precursor (a glicina) apresenta comportamento zwiteriônico, com separação de duas cargas em pH neutro, uma positiva no grupo amino e uma negativa no grupo fosfonato (COUTINHO e MAZO, 2005; AMARANTE JÚNIOR et al., 2002). Em razão de suas propriedades únicas, como degradação a produtos atóxicos, o glifosato (Figura 1) tem sido descrito como herbicida ideal (ARAÚJO, 2002).

\section{FIGURA 1 - FÓRMULA ESTRUTURAL DO HERBICIDA GLIFOSATO}<smiles>O=C(O)CNCP(=O)(O)O</smiles>

O grupo fosfonato R-PO $(\mathrm{OH})_{2}$ do composto tem a habilidade de formar complexos fortes com metais. Todos os processos de adsorção, fotodegradação e biodegradação dos fosfonatos são modificados pela presença de íons metálicos devido à formação de complexos solúveis e não-solúveis (COUTINHO e MAZO, 2005; AFONSO, HERSZAGE e AFONSO, 2001).

O herbicida apresenta (além do grupo fosfonato) os grupos funcionais amino e carboxilato que podem se coordenar fortemente com íons metálicos, especialmente os de transição, como Ni (II), Co(III), Zn(II), Fe(III), Cd(II) e Cu(II) (GLASS, 1984; DANIELE et al., 1997; PRENESTI e GULMINI, 1998; HEINEKE, FRANKLLIN e RAYMOND, 1994). A habilidade para se coordenar como ligante tridentado coloca o glifosato numa posição privilegiada entre os herbicidas (SUBRAMANIAM e HOGGARD, 1988; COUTINHO e MAZO, 2005).

O objetivo deste trabalho foi estudar o comportamento eletroquímico e espectrofotométrico do complexo $\mathrm{Cu}(\mathrm{II})$-glifosato, visando o desenvolvimento de metodologia para a análise direta desse herbicida.

\section{MATERIAL E MÉTODOS}

Todos os reagentes (grau analítico) foram utilizados sem prévia purificação, sendo as soluções preparadas com água purificada pelo sistema Milli-Q (Millipore Co). O glifosato utilizado foi cedido pela Milenia Agrociências (Londrina, PR).

Para o estudo espectrofotométrico utilizou-se espectrofotômetro Hitachi, modelo U-2010. As soluções foram preparadas com diferentes razões molares [glifosato]:[Cu(II)] $(0,3,0,5$ 0,8 e $1,0)$ e tiveram o pH justado para 5,5 com a adição de solução diluída de hidróxido de sódio. Foram realizadas varreduras nos comprimentos de onda entre 200 e 400 nm e entre 400 e $1000 \mathrm{~nm}$ nas regiões do ultravioleta (UV) e do visível, respectivamente.

No estudo eletroquímico empregou-se analisador polarográfico EG\&G PAR, modelo 394, e cela eletroquímica para três eletrodos. Usou-se eletrodo de gota suspensa de mercúrio (HMDE) como eletrodo de trabalho, fio de platina como eletrodo auxiliar e $\mathrm{Ag} / \mathrm{AgCl}$ saturado como eletrodo de referência. Os parâmetros utilizados na voltametria de onda quadrada (SWV) foram: amplitude de pulso $20 \mathrm{mv}$, incremento de varredura $2 \mathrm{mV}$ e freqüência $100 \mathrm{~Hz}$. Como eletrólito suporte usouse $0,1 \mathrm{~mol} \mathrm{~L}^{-1} \mathrm{KNO}_{3}(\mathrm{pH} \mathrm{5,5)}$. Antes de cada experimento a solução na cela eletroquímica foi deaerada com $\mathrm{N}_{2}$ durante 4 minutos. 


\section{RESULTADOS E DISCUSSÃO}

Para o estudo espectrofotométrico do complexo manteve-se fixa a concentração de $\mathrm{Cu}$ (II) e variou-se a de glifosato. As concentrações de $\mathrm{Cu}$ (II) utilizadas foram $4 \times 10^{-4} \mathrm{~mol} \mathrm{~L}^{-1} \mathrm{e} 8 \times 10^{-3} \mathrm{~mol} \mathrm{~L}^{-1}$ para as regiões do ultravioleta e do visível, respectivamente. As razões molares do complexo [glifosato]:[Cu(II)] foram 0,3, 0,5, 0,8 e 1,0.

As Figuras 2a e 3a apresentam os espectros da molécula de glifosato nas regiões do ultravioleta (UV) e do visível. Pode ser observado nessas figuras que o glifosato não apresenta bandas de absorção, uma vez que não contém grupos cromóforos em sua estrutura que absorvam em comprimentos de onda maiores que $200 \mathrm{~nm}$ (SATO et al., 2001). Assim, a detecção espectrofotométrica desse herbicida é possível somente com a derivação da molécula com a introdução de grupos capazes de absorver na região do UV (PICÓ, RODRIGUEZ e MANES, 2003; STALIKAS e KONIDARI, 2001). Contudo, pode-se utilizar metodologia de análise direta de glifosato (sem derivatização) mediante sua complexação com metais, tal como Cu(II) (COUTINHO et al., 2006).

\section{FIGURA 2 - ESPECTRO DA REGIÃO DO UV DO COMPLEXO Cu(II)-GLIFOSATO EM VÁRIAS RAZÕES MOLARES [GLIFOSATO]: [Cu(II)]}

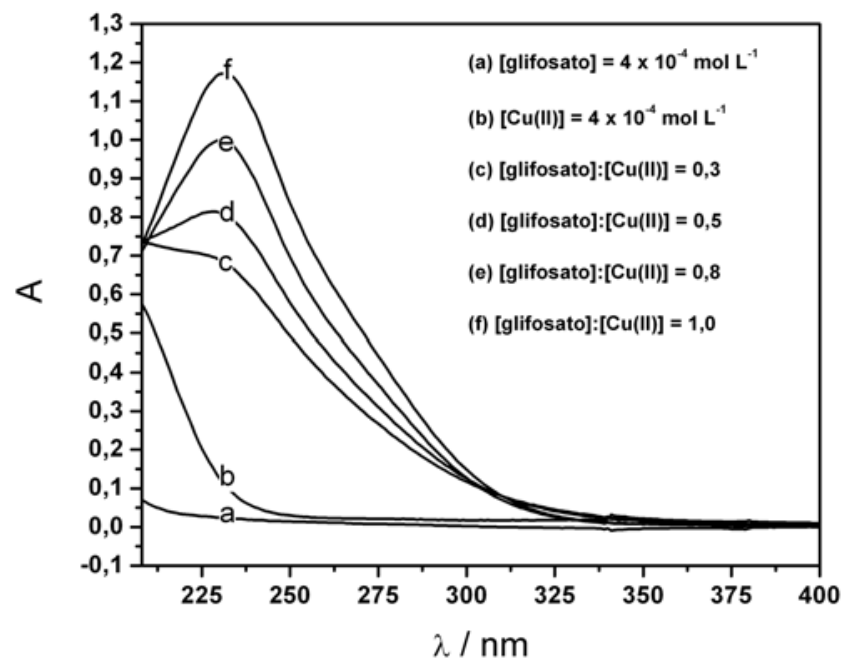

A Figura $2 \mathrm{~b}$ mostra que o $\mathrm{Cu}$ (II) não absorve na região entre 200 e $400 \mathrm{~nm}$. Todavia, quando foram adicionadas quantidades de glifosato na solução de $\mathrm{Cu}(\mathrm{II})$ (Figura $2 \mathrm{c}$ - 2f) verificou-se $\mathrm{o}$ aparecimento gradual de banda de absorção em aproximadamente $231 \mathrm{~nm}$. Essa banda foi atribuída à transferência de carga ligante-metal, resultante da transferência de um elétron do grupo doador da molécula de glifosato para o Cu(II). É provável que o par de elétrons do grupo amino esteja envolvido nesse processo (GLASS, 1984).

A solução de $\mathrm{Cu}$ (II) apresentou banda de absorção em $812 \mathrm{~nm}$ na ausência de glifosato (Figura 3), entretanto, a presença de glifosato (Figura 3c - 3f) deslocou esse valor para $731 \mathrm{~nm}$. Interpretou-se essa banda como uma transição d-d, típica de muitos complexos de Cu(II) (DANIELE et al., 1997; GLASS, 1984). A intensidade de absorção também foi alterada pela presença do herbicida, resultando num aumento de 4 vezes na intensidade da banda do $\mathrm{Cu}(\mathrm{II})$ não-complexado (Figura 3b).

Comparando os resultados obtidos neste trabalho com os dados apresentados na literatura (DANIELE et al., 1997; SHEALS, PEARSSON e HEDMAN, 2001) propõem-se que a molécula de glifosato no complexo $\mathrm{Cu}$ (II)-glifosato ocupa três das seis posições em um octaedro distorcido (Distorção de JahnTeller). A molécula de glifosato forma com o $\mathrm{Cu}$ (II) dois anéis quelatos de cinco membros orientados no plano equatorial (através dos grupos amino, carboxilato e o fosfonato). Completando esse octaedro ligamse três moléculas de água: uma fechando o plano equatorial e as outras duas ocupando os vértices do 
octaedro. A Figura 4 apresenta a estrutura molecular proposta para o complexo $\mathrm{Cu}(\mathrm{II})$-glifosato.

\section{FIGURA 3 - ESPECTRO DA REGIÃO DO VISÍVEL DO COMPLEXO CU(II)-GLIFOSATO EM VÁRIAS RAZÕES MOLARES [GLIFOSATO]:[Cu(II)]}

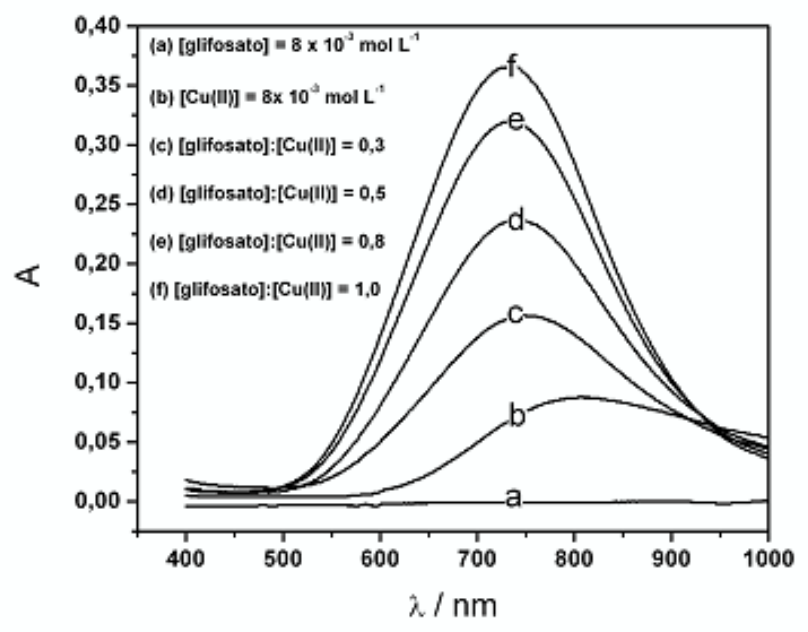

FIGURA 4 - ESTRUTURA MOLECULAR DO COMPLEXO Cu(II)-GLIFOSATO<smiles>CC1(C)OC(=O)CN2CC(=O)OCC21</smiles>

No estudo eletroquímico do complexo Cu(II)-glifosato utilizou-se a técnica de voltametria de onda quadrada SWV (do inglês square wave voltammetry), que constitui uma das (técnicas voltamétricas de pulso) mais rápidas e sensíveis. Quando se considera o uso de eletrodo de mercúrio (HMDE), a maior vantagem da SWV é a possibilidade de se promover varredura completa do potencial durante o tempo de vida de uma única gota de mercúrio. Isso reduz consideravelmente o tempo necessário para a realização do experimento (DE SOUZA, MACHADO e AVACA, 2003; DE SOUZA et al., 2004).

O glifosato não apresenta resposta eletroquímica em eletrodo de HMDE, ou seja, é polarograficamente inativo (TEÓFILO et al., 2004). Contudo, esse herbicida pode ser detectado na forma de complexo metálico mediante redução eletroquímica do metal presente no complexo (Cu(II) para $\mathrm{Cu}(0)$ nesse caso), como mostrado na Figura 5.

Como pode ser observado na Figura 5, o Cu(II) na ausência de glifosato apresentou potencial de pico $\mathrm{E}_{\mathrm{p}}$ igual a $0,034 \mathrm{~V}$. Entretanto, quando se adicionou concentração de glifosato equivalente a metade da concentração de $\mathrm{Cu}(\mathrm{II})$ (razão [glifosato]: $[\mathrm{Cu}(\mathrm{II})]=0,5$ ) apareceu novo pico com $\mathrm{E}_{\mathrm{p}}$ em aproximadamente -0,200 V e ocorreu diminuição na intensidade da corrente de pico do Cu(II) não-complexado. Para a razão molar igual a 0,5 existiram dois picos voltamétricos, um referente ao $\mathrm{Cu}(\mathrm{II})$ não-complexado $\left(\mathrm{E}_{\mathrm{p}}=0,034 \mathrm{~V}\right)$ 
e o outro referente ao $\mathrm{Cu}(\mathrm{II})$ complexado pelo glifosato $\left(\mathrm{E}_{\mathrm{p}}=-0,200 \mathrm{~V}\right)$. No voltamograma da solução que continha razão molar [glifosato]:[Cu(II)] = 1 observou-se que o pico referente ao $\mathrm{Cu}(\mathrm{II})$ não-complexado desapareceu, e que a intensidade da corrente de pico referente ao complexo aumentou. Pode-se dizer que nessa razão molar todo $\mathrm{Cu}$ (II) encontra-se na forma complexada.

\section{FIGURA 5 - VOLTAMOGRAMAS DE ONDA QUADRADA (SWV) DOS COMPLEXOS CU(II)-GLIFOSATO}

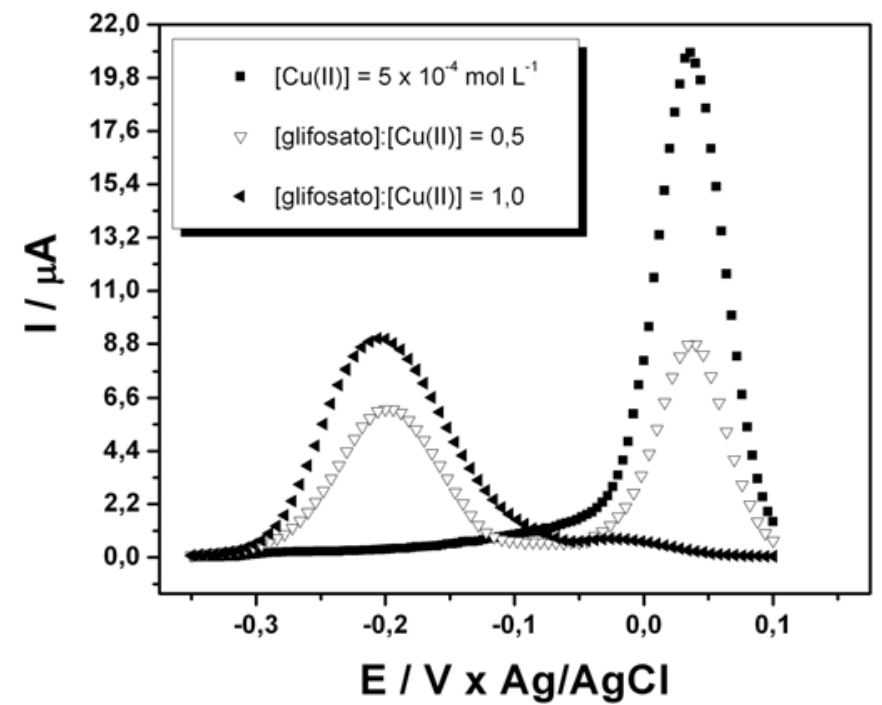

Conhecendo os potenciais de pico $\mathrm{E}_{\mathrm{p}}$ do $\mathrm{Cu}(\mathrm{II})$ complexado e não-complexado pode-se estimar a constante de estabilidade condicional do complexo, utilizando a equação de Nernst (SKOOG, HOLLER e NIEMAN, 1998):

$$
\left(E_{p}\right)_{C}-\left(E_{p}\right)_{M}=\frac{-0,0592}{n} \log K
$$

Em que:

$\left(E_{p}\right)_{C}=$ potencial de pico do complexo;

$\left(E_{p}\right)_{M}=$ potencial de pico do metal não-complexado;

$n=$ número de elétrons envolvidos no processo; e

$K=$ constante de estabilidade condicional do complexo.

Substituindo os termos pelos valores encontrados neste trabalho obteve-se a constante de estabilidade condicional do complexo na razão molar $\mathrm{Cu}(\mathrm{II})$-glifosato igual a log $K=7,9$. MORRILLO et al. (1994) utilizaram eletrodo de gota suspensa de mercúrio (HMDE) e voltametria de redissolução anódica e determinaram a constante de estabilidade condicional do complexo (na presença de excesso de glifosato) igual a $\log K=4,1$. Tais autores não determinaram o $\log K$ na razão molar 1:1 $\mathrm{Cu}(\mathrm{II})$ :glifosato.

O estudo inicial mostrou que o glifosato e o $\mathrm{Cu}(\mathrm{II})$ formam complexo forte e estável e que essa reação de complexação pode ser utilizada para a determinação de glifosato, sem a necessidade de derivatização. Futuramente, este estudo será estendido para outros metais, como $\mathrm{Ni}(\mathrm{II})$ e Co(II). 


\section{CONCLUSÃO}

O estudo das propriedades complexantes do glifosato pode ser justificada não somente pelo interesse químico, mas também porque as informações obtidas são essenciais para a compreensão do comportamento desse herbicida no solo e nas plantas.

O uso de metodologias que não necessitem de derivatização é muito interessante, pois resulta em economia de reagentes e de tempo no preparo das amostras. Desse modo, a habilidade do glifosato em complexar com grande variedade de metais pode ser utilizada com propósitos analíticos, como o desenvolvimento de metodologias de análise (voltamétrica e espectrofotométrica) desse herbicida em amostras de solo e água.

\section{ABSTRACT}

\section{VOLTAMMETRIC AND SPECTROPHOTOMETRIC STUDY OF CU(II)-GLYPHOSATE COMPLEX}

The objective of this paper was to study the voltammetric and spectrophotometric behavior of $\mathrm{Cu}(\mathrm{II})$-glyphosate complex in solution. This complex presented two absorption bands in the ultraviolet-visible range (200 to $1000 \mathrm{~nm}$ ): one at $231 \mathrm{~nm}$ (charge-transfer) and other at $731 \mathrm{~nm}$ (electronic $\mathrm{d}$-d transition band). The voltammetric study was carried out using the square wave voltammetry (SWV), and it was observed that the presence of glyphosate displaced the potential of $\mathrm{Cu}(\mathrm{II})(+0.034 \mathrm{~V})$ to more negative value $(-0.2 \mathrm{~V})$. From these potential values, the conditional stability constant of the complex can be calculated by $\log K=7.9$. The results obtained in this paper, showed that the glyphosate can be detected in the complex form, without derivatization. The use of methodologies for direct analysis is very interesting, due the economy of reagents and time in the samples preparation. Thus, the ability of the glyphosate to form complex with a variety of metals, can be used with analytical purposes, as the development of methodologies of direct analysis (voltammetric and spectrophotometric) of this herbicide, in soil and water samples.

KEY-WORDS: CU(II); GLYPHOSATE; SPECTROPHOTOMETRY; VOLTAMMETRY.

\section{REFERÊNCIAS}

1 AFONSO, B. C.; HERSZAGE, J.; AFONSO, M. S. Iron(II)-phosphonate complexes. Polyhedron, v. 20, p. 1821-1830, 2001.

2 ARAÚJO, A. S. F. Biodegradação, extração e análise de glifosato em dois tipos de solos. Piracicaba, 2002. 83 p. Dissertação (Mestrado em Agronomia) - Escola Superior de Agricultura "Luiz de Queiroz", Universidade de São Paulo.

3 AMARANTE JÚNIOR, O. P.; SANTOS, T. C. R.; BRITO, N. M.; RIBEIRO, M. L. Glifosato: propriedades, toxicidade, usos e legislação. Química Nova, v. 25, n.4, p.589-593, 2002.

4 COUTINHO, C. F. B.; MAZO, L. H. Complexos metálicos com o herbicida glifosato: revisão. Química Nova, v. 28, n. 6, p. 1038-1045, 2005.

5 COUTINHO, C. F. B.; TANIMOTO, S. T.; GALLI, A.; GARBELLINI, G. S.; TAKAYAMA, M.; DO AMARAL, R. B.; MAZO, L. H.; MACHADO, S. A. S.; AVACA, L. A. Pesticidas: mecanismo de ação, degradação e toxidez. Pesticidas: revista de ecotoxicologia e meio ambiente, v. 15, p. 65-72, 2005.

6 COUTINHO, C. F. B.; SILVA, M. O.; MAZO, L. H.; MACHADO, S. A. S. Influence of glyphosate on the copper dissolution in phosphate buffer. Appl. Surf. Sci., in press, 2006.

7 DANIELE, P. G.; STEFANO, C.; PRENESTI, E.; SAMMARTANO, S. Copper(II) complexes of N(phosphonomethyl)glycine in aqueous solution: a thermodynamic and spectrophotometric study. Talanta, v. 45, n. 2 , p. 425-431, 1997.

8 DE SOUZA, D.; MACHADO, S. A. S.; AVACA, L. A. Voltametria de onda quadrada. Primeira parte: aspectos teóricos. Química Nova, v. 26, n. 1, p. 81-89, 2003. 
9 DE SOUZA, D.; CODOGNOTO, L.; MALAGUTTI, A. R.; TOLEDO, R. A.; PEDROSA, V. P.; OLIVEIRA, R. T. S.; MAZO, L. H.; MACHADO, S. A. S.; AVACA, L. A. Voltametria de onda quadrada. Segunda parte: aplicações. Química Nova, v. 27, n. 5, p. 790-797, 2004.

10 GLASS, R. L. Metal-complex formation by glyphosate. J. Agric. Food Chem., v.32, n.6, p. 1249-1253, 1984.

11 HEINEKE, D.; FRANKLLIN, S. J.; RAYMOND, K. N. Coordination chemistry of glyphosate: structural and spectroscopic characterization of Bis(glyphosate)metal(III) complexes. Inorg. Chem., v. 33, n. 11, p. 2413-2421, 1994.

12 MORILLO, E.; MAQUEDA, C.; BEJARANO, M.; MADRID, L.; UNDABEYTIA, T. Cu(II)-glyphosate system: a study by anodic stripping voltammetry and the influence on $\mathrm{Cu}$ adsorption by montmorillonite. Chemosphere, v. 28, n. 12, p. 2185-2196, 1994.

13 PICÓ, Y.; RODRIGUEZ, R.; MAÑES, J. Capillary electrophoresis for the determination of pesticide residues. Trends Anal. Chem., v. 22, n. 3, p. 133-151, 2003.

14 PRENESTI, E.; GULMINI, M. Nickel(II) complexes of N-(phosphonomethyl)glycine in aqueous solutions. Ann. Chim., v. 88, p. 591-595, 1998.

15 SATO, K.; JIN, J. Y.; TAKEUCHI, T.; MIWA, T.; SUENAMI, K.; TAKEKOSHI, Y.; KANNO, S. Integrated pulsed amperometric detection of glufosinate, bialaphos and glyphosate at gold electrode in anion-exchange chromatography. J. Chromatogr. A, v. 919, p. 313-320, 2001.

16 SHEALS, J.; PEARSSON, P.; HEDMAN. B. IR and EXAFS spectroscopic studies of glyphosate protonation and copper(II) complexes of glyphosate in aqueous solution. Inorg. Chem., v. 40, n. 17, p. 4302-4309, 2001.

17 SKOOG, D. A.; HOLLER, F. J.; NIEMAN, T. A. Principles of instrumental analysis. $5^{\text {th }}$ ed. Orlando: Saunders College Publishing, 1998. p. 570-579.

18 STALIKAS, C. D.; KONIDARI, C. N. Analytical methods to determine phosphonic and amino acid groupcontaining pesticides. J. Chromatogr. A, v. 907, p. 1-19, 2001.

19 SUBRAmANiAM, V.; HOGGARD, P. E. Metal complexes of glyphosate. J. Agric. Food Chem., v. 36, n. 6, p. 1326-1329, 1988.

20 TEÓFILO, R. F.; REIS, E. L.; REIS, C.; SILVA, G., A.; KUBOTA, L. T. Experimental design employed to square wave voltammetry response optimization for the glyphosate determination. J. Braz. Chem. Soc., v. 15, n. 6, p. 865-871, 2004.

\section{AGRADECIMENTOS}

Os autores agradecem a CAPES, Milenia Agrociências e ao IQSC-USP. 